\title{
Heterotopic caesarean section scar pregnancy after embryo transfer: a case report successfully treated with aspiration
}

\author{
Marcelo Lucchesi Montenegro; Rodrigo Furtado²; Carolina Westphalen Savari; Francisco Furtado Filho \\ ${ }^{1}$ Fertway Fertility Clinic, Curitiba, Parana, Brazil. \\ 2 Mackenzie Evangelical College of Paraná (FEMPAR), Curitiba, Parana, Brazil.
}

\begin{abstract}
Heterotopic caesarean section scar pregnancy (HCSP) is a rare presentation of an ectopic pregnancy with no standard treatment proposed due to the low number of cases. Besides, it faces the challenge of preserving the concurrent intrauterine pregnancy. The early diagnose and conservative management is mandatory in order to preserve fertility and to prevent catastrophic outcomes to the mother. We present a 35-year-old Brazilian woman submitted to an embryo transfer followed by a HCSP. In order to spare the concurrent intrauterine pregnancy, we performed an embryo reduction of the ectopic gestational sac by ultrasound guided aspiration on $6^{\text {th }}$ week of gestation. The patient gave birth to a healthy baby on $39^{\text {th }}$ week. With this case report we intend to review similar cases described previously and discuss the best options available for management of this complex situation.
\end{abstract}

Keywords: Caesarean scar pregnancy, embryo aspiration, heterotopic pregnancy, selective embryo reduction

\section{Introduction}

Ectopic pregnancies (EP) occur, by definition, after embryo implantation on an anomalous location. The rarest form of an EP happens when the implantation occurs on the scar of a previous caesarean section embedded in local myometrium. The incidence of this event is estimated between 1:1688 to 1:2216 pregnancies..$^{1-3}$ If they are not identified at early stages, they can be associated with serious complications, such as extensive haemorrhages or uterine rupture, resulting in possibly catastrophic maternal and fetal outcomes. ${ }^{4}$ Thus, early diagnosis by transvaginal ultrasound (TVUS) is essential to enable conservative treatment and fertility preservation. ${ }^{5}$

Even more uncommon, heterotopic pregnancies occur when one embryo is normoimplanted and another embryo is implanted on an ectopic location, which can be on a previous caesarean section scar. Only a few cases of heterotopic caesarean section scar pregnancy (HCSP) have been reported, being the first published in 2003. ${ }^{6}$ Due to its rarity, the incidence of this condition has not been established. Therefore, there are no defined protocols about management to guide treatment. Thus, different attempts have been described, varying from expectant management to clinical or surgical intervention - ultrasound guided aspiration or infusion of potassium chloride or methotrexate and hysterectomy. ${ }^{7,8}$

On this report, we intend to describe a HCSP resulting from a double embryo transfer treated successfully by embryo reduction performed by ultrasound guided aspiration of the ectopic gestational sac. On this case, the patient evolved to a normal gestation up to term, when a healthy baby was delivered. By presenting our experience and results, we aim to review previous similar cases and discuss the best option for management of future occurrences.

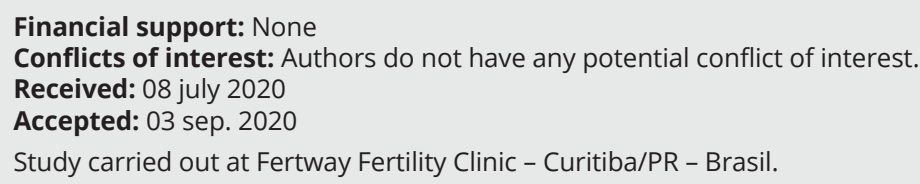

Copyright Montenegro et al. This is an Open Access article distributed under the terms of the Creative Commons Attribution License, which permits unrestricted use, distribution, and reproduction in any medium, provided the original work is properly cited. 


\section{Case Report}

A 35-year-old Brazilian woman, from Curitiba (Parana), gravida 4, para 1 and 3 previous abortions, was admitted on a fertility clinic because of her 8 years history of secondary infertility. She had been previously submitted to in vitro fertilization (IVF) treatments that resulted in three miscarriages and one pregnancy delivery by elective caesarean section at the $39^{\text {th }}$ week four years ago. Besides infertility, no other particularities were identified on her medical records. Once she desired a second child, a further cycle of ovarian stimulation and IVF was proposed.

After pre-treatment with oral combined contraceptive, she was submitted to a controlled ovarian stimulation with recombinant FSH + hMG (300 UI/day); a series of TVUS was performed to follow follicles development; as soon as the leading follicle achieved $14 \mathrm{~mm}$ of diameter, a GnRH antagonist was added (Cetrotide $0.25 \mathrm{mg} / \mathrm{day}$ ). On the tenth day of stimuli, hCG was administered (Ovidrel 500UI) to trigger ovulation and, 36 hours later, 6 mature oocytes were retrieved. After intracytoplasmic sperm injection (ICSI), 3 blastocysts were cultivated until day 5. Genetic analysis of trophectoderm cells by next generation sequencing (NGS) test demonstrated 3 euploid embryos.

On the following month, endometrium was prepared with estradiol $6 \mathrm{mg} /$ day for 12 days plus micronized progesterone $600 \mathrm{mg} /$ day on 5 extra days. Two embryos were thawed and transferred on D5 guided by transabdominal ultrasound. Eleven days after the procedure, pregnancy test was positive.

Four weeks after transfer, TVUS evidenced presence of an intrauterine gestational sac with one live embryo and a second ectopic gestational sac implanted on previous caesarean section scar also filled with an embryo with cardiac heartbeat present. Both embryos had a crown-rump length compatible with 6 weeks plus 1 day. Patient remained asymptomatic, without any vaginal discharge or bleeding and with stable vital signs.

Owing its abnormal location and non-evolving aspect of the ectopic pregnancy, it was opted to perform an embryo reduction of the unviable gestational sac as soon as possible, in order to reduce maternal and fetal risks. Consent forms were signed by the couple. When the gestational age was 6 weeks +5 days, an aspiration puncture of the ectopic gestational sac was performed, guided by TVUS, over slight sedation (intravenous propofol). An oocyte retrieval needle of 16 gauges was used to aspirate the content, without any undesirable intercurrences (Figure 1).

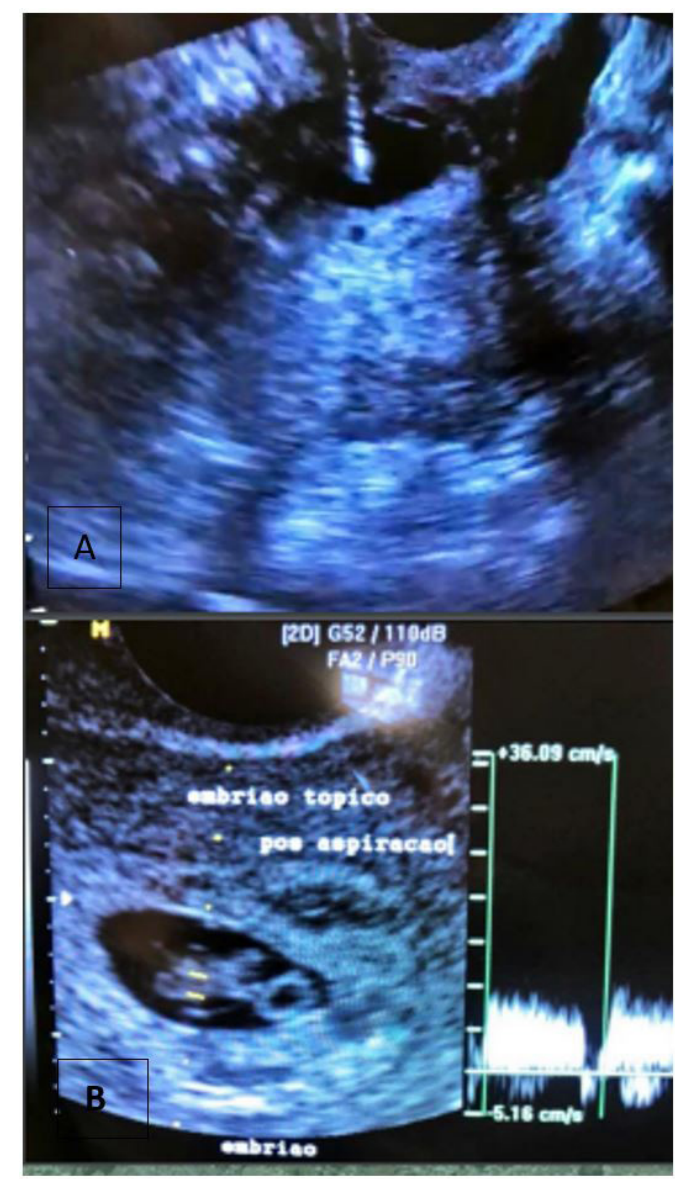

Figure 1. Ultrasound image showing the moment of gestational sac aspiration with a puncture needle (A) and intrauterine concurrent embryo with normal heartbeat short after the procedure was finished (B). 
Three day later, a control TVUS evidenced one topic gestational sac implanted on uterine fundus with a viable embryo and, on the anterior uterine wall, at the level of uterine isthmus, a heterogenic and vascularized image was seen, interpreted as ovular remains of the reduced ectopic pregnancy. This image was visible by ultrasound until $35^{\text {th }}$ week of gestation, when a rounded amorphous nodule was expelled vaginally.

Pre-natal care had no other problems and the patient was taken to a second elective caesarean section delivery at 39 weeks and 3 days of pregnancy. A healthy male baby was born weighing 3775 grams.

\section{Discussion}

The current case illustrates a HCSP successfully managed. There are only a few similar reports described in previous literature. The first report of this rare pathology was published in $2003,{ }^{6}$ and was treated successfully with ultrasound guided potassium chloride $(\mathrm{KCl})$ injection inside cardiac area of the ectopic embryo. Since then, some other treatments were proposed and instituted.

On a review from 2016, it was demonstrated other 23 similar pregnancies since then, approached on a varied gestational age, as soon as 5 weeks until one case treated at 16 weeks of gestation (Table 1). ${ }^{8}$ Seven patients were submitted to expectant treatment - from them, four delivered live babies. Therefore, this approach emerged as an alternative, especially when the ectopic pregnancy has non-viable characteristics (i.e., absence of cardiac heartbeat). The remaining 16 patients were referred to embryo reduction procedures, which can be considered the most suitable when embryonic heartbeat is identified. $\mathrm{KCl}$ injection was done in 10 cases; surgical removal of the gestational sac in 3 and TVUS guided aspiration also in 3 patients; those treatments resulted in 9, 3 and 2 alive babies' births of the concurrent embryo, respectively.

Other case reports were also published describing similar situations. For instance, it was described the treatment of a triplet pregnancy where one of the embryos was located on caesarean scar and was eliminated by aspiration on

Table 1. Summary of previous heterotopic caesarean section scar pregnancy cases reported

\begin{tabular}{|c|c|c|c|c|c|c|c|c|c|}
\hline 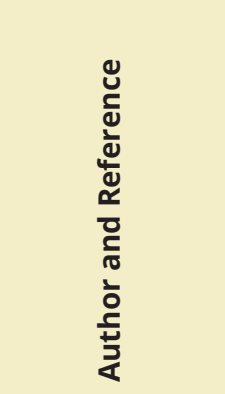 & $\underset{0}{\stackrel{0}{\alpha}}$ & $\begin{array}{l}\text { 든 } \\
\text { 음 } \\
\text { 등 }\end{array}$ & 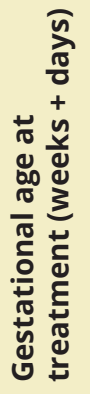 & 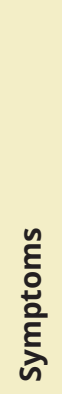 & 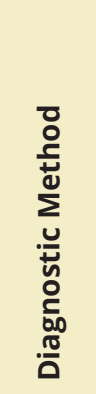 & 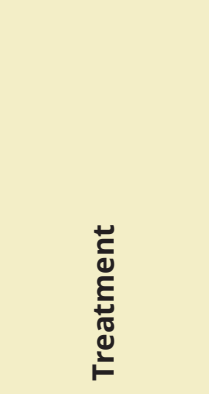 & 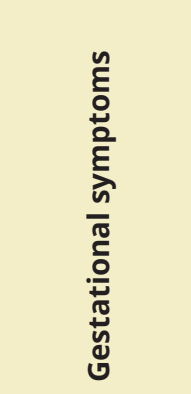 & 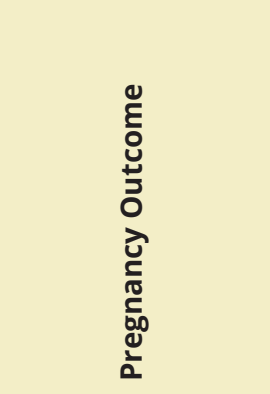 & 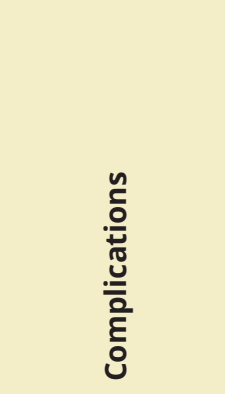 \\
\hline Salomon et al. ${ }^{6}$ & 36 & IVF & 8 & No & TVUS & $\mathrm{KCl}$ injection & No & $\begin{array}{l}\text { Live birth by } \\
\text { C-section at } 36 \mathrm{wk} \\
\text { due to PROM }\end{array}$ & No \\
\hline Wang et al. ${ }^{9}$ & 38 & IVF & 10 & No & TVUS & $\mathrm{KCl}$ injection & No & $\begin{array}{l}\text { Live birth by CD } \\
\text { at } 35 \text { wk due to } \\
\text { preterm labor }\end{array}$ & $\begin{array}{l}\text { Bilateral } \\
\text { internal iliac } \\
\text { artery ligation } \\
\text { and blood } \\
\text { transfusion } \\
\text { due to massive } \\
\text { bleeding }\end{array}$ \\
\hline Litwickaet al. ${ }^{10}$ & 31 & IVF & 8 & No & TVUS & $\begin{array}{c}\mathrm{KCl} \text { and } \\
\text { Methotrexate } \\
\text { injection }\end{array}$ & $\begin{array}{c}\text { Vaginal } \\
\text { bleeding } \\
\text { and uterine } \\
\text { contractions }\end{array}$ & $\begin{array}{l}\text { Live birth by } \\
\text { C-section at } 36 \text { wk } \\
\text { due toplacental } \\
\text { abruption }\end{array}$ & $\begin{array}{l}\text { Birth defect } \\
\text { and blood } \\
\text { transfusion } \\
\text { due to blood } \\
\text { loss }\end{array}$ \\
\hline Hsieh et al..11 & 38 & IVF & 6 & No & TVUS & $\begin{array}{l}\text { TVUS guided } \\
\text { embryo } \\
\text { aspiration }\end{array}$ & No & $\begin{array}{l}\text { Live birth by } \\
\text { C-section at } 36 \mathrm{wk} \\
\text { due to preterm } \\
\text { labor }\end{array}$ & No \\
\hline
\end{tabular}

In vitro fertilization (IVF); transvaginal ultrasound (TVUS); transabdominal ultrasound (TAUS); weeks (WK); potassium chloride (KCl); premature rupture of membranes (PROM). 
Table 1. Continued...

\begin{tabular}{|c|c|c|c|c|c|c|c|c|c|}
\hline 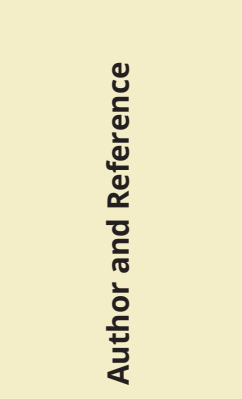 & $\stackrel{0}{4}$ & 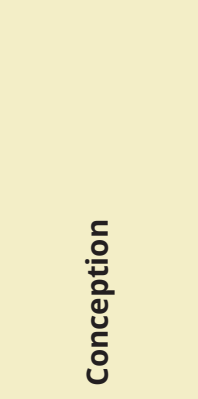 & 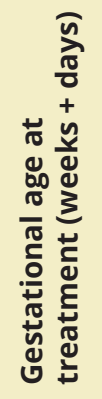 & है & 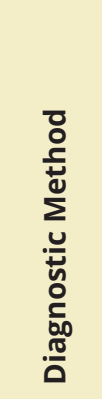 & 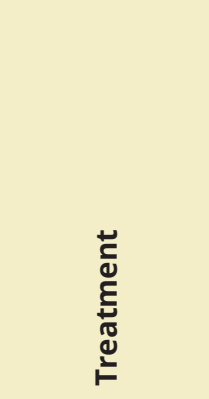 & 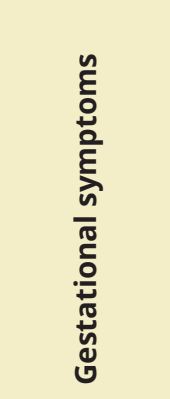 & 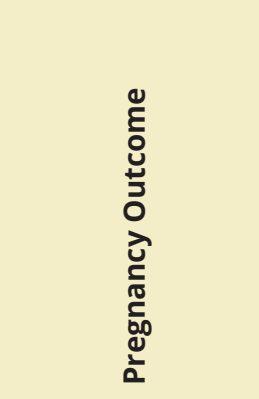 & 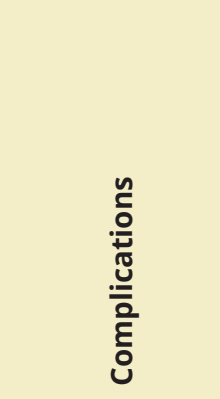 \\
\hline Demirel et al. ${ }^{12}$ & 34 & Spontaneous & $6+5$ & $\begin{array}{l}\text { Vaginal } \\
\text { bleeding }\end{array}$ & TVUS & $\begin{array}{l}\text { Laparoscopic } \\
\text { Excision }\end{array}$ & No & $\begin{array}{c}\text { Live birth by } \\
\text { C-section at } 32 \text { wk }\end{array}$ & No \\
\hline Gupta et al..$^{13}$ & 37 & IVF & $6+3$ & No & TVUS & $\begin{array}{l}\text { TVUS guided } \\
\text { embryo } \\
\text { aspiration }\end{array}$ & - & $\begin{array}{c}\text { Pregnancy } \\
\text { terminations at } 12 \\
\text { wk due to trisomy } \\
13\end{array}$ & No \\
\hline Bai et al. ${ }^{14}$ & 37 & IVF & $7+6$ & $\begin{array}{l}\text { Vaginal } \\
\text { bleeding }\end{array}$ & TVUS & $\begin{array}{c}\text { Expectant } \\
\text { management }\end{array}$ & No & $\begin{array}{l}\text { Live birth by } \\
\text { C-section at } 36 \text { wk } \\
+4 \text { days due to } \\
\text { preterm labor }\end{array}$ & No \\
\hline Uysal and Uysal'15 & 29 & Spontaneous & 8 & No & TVUS & $\mathrm{KCl}$ injection & No & $\begin{array}{c}\text { Live birth by } \\
\text { C-section at } 35 \mathrm{wk} \\
\text { due to preterm } \\
\text { labor }\end{array}$ & No \\
\hline Yazicioglu et al. ${ }^{16}$ & 23 & Spontaneous & $7+2$ & $\begin{array}{c}\text { Vaginal } \\
\text { bleeding }\end{array}$ & TVUS & $\mathrm{KCl}$ injection & No & $\begin{array}{l}\text { Live birth by } \\
\text { C-section at } 30 \text { wk } \\
+3 \text { days due to } \\
\text { PROM }\end{array}$ & No \\
\hline Jurkovic et al. ${ }^{1}$ & 36 & Spontaneous & 7 & No & TVUS & $\mathrm{KCl}$ injection & No & $\begin{array}{c}\text { Live birth by } \\
\text { C-section at } 31 \text { wk }\end{array}$ & No \\
\hline Wang et al. ${ }^{9}$ & 31 & IVF & 7 & $\begin{array}{l}\text { Vaginal } \\
\text { bleeding }\end{array}$ & TVUS & $\begin{array}{l}\text { Hysteroscopic } \\
\text { excision }\end{array}$ & No & $\begin{array}{c}\text { Live birth by } \\
\text { C-section at } 39 \text { wk }\end{array}$ & No \\
\hline Taşkin et al. ${ }^{17}$ & 24 & Spontaneous & $8+4$ & $\begin{array}{l}\text { Vaginal } \\
\text { bleeding }\end{array}$ & TVUS & $\mathrm{KCl}$ injection & $\begin{array}{l}\text { Vaginal } \\
\text { bleeding }\end{array}$ & $\begin{array}{c}\text { Live birth by } \\
\text { C-section at } 34 \text { wk } \\
\text { due to preterm } \\
\text { labor }\end{array}$ & $\begin{array}{c}\text { Blood } \\
\text { transfusion } \\
\text { due to massive } \\
\text { bleeding }\end{array}$ \\
\hline $\begin{array}{c}\text { Dueñas-Garcia } \\
\text { and Young }{ }^{18}\end{array}$ & 34 & Spontaneous & 5 & No & $\begin{array}{l}\text { TVUS; } \\
\text { TAUS; } \\
\text { MRI }\end{array}$ & $\begin{array}{c}4 \text { doses of } \\
\text { intramuscular } \\
\text { methotrexate }\end{array}$ & No & $\begin{array}{l}\text { Pregnancy } \\
\text { termination }\end{array}$ & $\begin{array}{l}\text { Induced } \\
\text { abortion }\end{array}$ \\
\hline Ugurlucan et al..$^{19}$ & 34 & IVF & 6 & No & TVUS & $\begin{array}{l}\text { KCl injection + } \\
\text { TVUS guided } \\
\text { embryo } \\
\text { aspiration }\end{array}$ & $\begin{array}{l}\text { Placenta } \\
\text { previa and } \\
\text { placenta } \\
\text { accreta }\end{array}$ & $\begin{array}{c}\text { Live birth by } \\
\text { C-section at } 34 \text { wk }\end{array}$ & $\begin{array}{c}\text { Bilateral } \\
\text { internal iliac } \\
\text { artery ligation } \\
\text { + subtotal } \\
\text { hysterectomy } \\
\text { due to massive } \\
\text { bleeding }\end{array}$ \\
\hline Lui et al. ${ }^{20}$ & 36 & $\begin{array}{l}\text { Intrauterine } \\
\text { insemination }\end{array}$ & $\begin{array}{c}5 \text { and } \\
5+3\end{array}$ & No & TVUS & $\begin{array}{l}\text { Consecutive } \\
\text { TVUS guided } \\
\text { embryo } \\
\text { aspiration }\end{array}$ & No & $\begin{array}{c}\text { Live birth by } \\
\text { C-section at } 37 \text { wk }\end{array}$ & $\begin{array}{c}\text { Elective } \\
\text { embolization of } \\
\text { uterine artery } \\
\text { due to massive } \\
\text { bleeding }\end{array}$ \\
\hline
\end{tabular}

In vitro fertilization (IVF); transvaginal ultrasound (TVUS); transabdominal ultrasound (TAUS); weeks (WK); potassium chloride (KCI); premature rupture of membranes (PROM). 
Table 1. Continued...

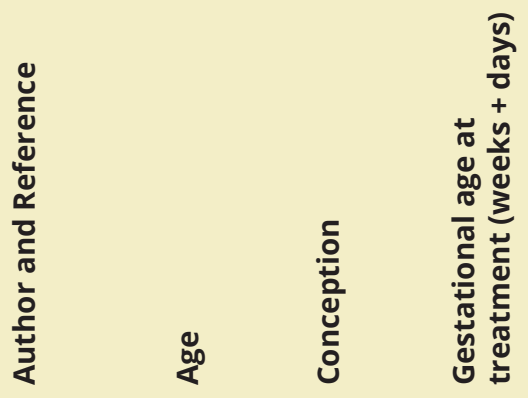

Kim et al. ${ }^{21}$

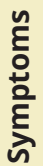

है

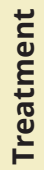

Armbrust et al. ${ }^{22} \quad 36$

No
IVF
Laparoscopic excision

No

है

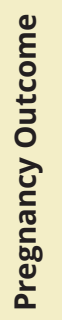
accreta

Placenta previa and placenta acrcreta

Abdominal

Ouyang et al. ${ }^{3}$

IVF

$6+5$ pain and Vaginal bleeding

Ouyang et al. ${ }^{3}$

IVF

$5+6$

Abdominal pain

TVUS

Abdominal

Ouyang et al. ${ }^{3} \quad 38$

IVF pain

TVUS

Ouyang et al. ${ }^{3} \quad 28$

IVF

$6+3 \quad$ No

TVUS

$6+3$

Vagina bleeding

Abdominal

Ouyang et al. ${ }^{3} \quad 34$

IVF management

Expectant management

TVUS

$\mathrm{KCl}$ injection

\section{Expectant management \\ Placenta
previa}

-

TVUS
Expectant management pain and

Vaginal bleeding
Live birth by C-section at $37 \mathrm{wk}$ +3 days

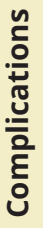

Bilateral internal iliac artery ligation + subtotal hysterectomy due to massive bleeding

Live birth by C-section at $37 \mathrm{wk}$

No

Subtotal hysterectomy and blood transfusion due to uterine rupture and massive bleeding

Massive bleeding due to placenta previa

Live birth by

Pregnancy termination at 13 wk

Spontaneous abortion

Live birth by C-section at 36 wk due to PROM

Spontaneous abortion

$$
\text { Ongoing }
$$

pregnancy at 18 wk when case reported

In vitro fertilization (IVF); transvaginal ultrasound (TVUS); transabdominal ultrasound (TAUS); weeks (WK); potassium chloride (KCI); premature rupture of membranes (PROM). 
the first trimester, followed by birth of two babies on the $32^{\text {nd }}$ week. ${ }^{11}$ The use of TVUS was proposed later. A HSCP was treated with the aid of TVUS aspiration on the United Kindom in 2010; unfortunately, even though the procedure successfully kept the intrauterine embryo alive, further investigation demonstrated trisomy 13 on the remaining one, thus its suction on $12^{\text {th }}$ week was also performed..$^{13}$ Finally, in 2014, a group from Hong Kong executed the first successful aspiration of the ectopic gestational sac of a HSCP, also resulting in healthy pregnancy of the concurrent baby, which was delivered at term..$^{20}$

EP occurred more frequently after assisted reproductive techniques (ART). ${ }^{23}$ Besides, embryo transferred performed on the third day of embryo development (D3) and the use of GnRH agonist as an ovulation trigger were associated with higher incidences of EP..$^{24,25}$ Interestingly, neither of those features were present on the case described previously.

As seen, the case presented here is the first HSCP reported in Brazil and the third successfully treated by aspiration of the ectopic sac followed by a live birth of the remaining embryo. Even though the low number of cases still do not allow the definition of a protocol, a trend can be established by this review. Expectant management can be an option in cases when embryonic heartbeat is not identified and some embryo reduction method must be performed if cardiac activity is observed on the ectopic embryo. Our experience and previous literature review support that TVUS guided aspiration is a safe and effective method that can be used to treat HSCP.

\section{References}

1. Jurkovic D, Hillaby K, Woelfer B, Lawrence A, Salim R, Elson C. First-trimester diagnosis and management of pregnancies implanted into the lower uterine segment Cesarean section scar. Ultrasound Obstet Gynecol. 2003;21(3):220-7. http://dx.doi.org/10.1002/ uog.56. PMid:12666214.

2. Seow K-M, Huang L-W, Lin Y-H, Yan-Sheng Lin M, Tsai Y-L, Hwang J-L. Cesarean scar pregnancy: issues in management. Ultrasound Obstet Gynecol. 2004;23(3):247-53. http://dx.doi.org/10.1002/uog.974. PMid:15027012.

3. Ouyang Y, Li X, Yi Y, Gong F, Lin G, Lu G. First-trimester diagnosis and management of Cesarean scar pregnancies after in vitro fertilization-embryo transfer: a retrospective clinical analysis of 12 cases. Reprod Biol Endocrinol. 2015;13(1):126. http://dx.doi. org/10.1186/s12958-015-0120-2. PMid:26589452.

4. Lai YM, Lee J, Lee C, Chen T, Soong Y. An ectopic pregnancy embedded in the myometrium of a previous cesarean section scar. Acta Obstet Gynecol Scand. 1995;74(7):573-6. http://dx.doi.org/10.3109/00016349509024394. PMid:7618461.

5. Vial Y, Petignat P, Hohlfeld P. Pregnancy in a Cesarean scar. Ultrasound Obstet Gynecol. 2000;16(6):592-3. http://dx.doi. org/10.1046/j.1469-0705.2000.00300-2.x. PMid:11169360.

6. Salomon LJ, Fernandez H, Chauveaud A, Doumerc S, Frydman R. Successful management of a heterotopic Caesarean scar pregnancy: potassium chloride injection with preservation of the intrauterine gestation: case report. Hum Reprod. 2003;18(1):18991. http://dx.doi.org/10.1093/humrep/deg010. PMid:12525465.

7. OuYang Z, Yin Q, Xu Y, Ma Y, Zhang Q, Yu Y. Heterotopic cesarean scar pregnancy: diagnosis, treatment, and prognosis. J Ultrasound Med. 2014;33(9):1533-7. http://dx.doi.org/10.7863/ultra.33.9.1533. PMid:25154932.

8. Yu H, Luo H, Zhao F, Liu X, Wang X. Successful selective reduction of a heterotopic cesarean scar pregnancy in the second trimester: a case report and review of the literature. BMC Pregnancy Childbirth. 2016;16(1):380. http://dx.doi.org/10.1186/ s12884-016-1171-x. PMid:27894281.

9. Wang CJ, Tsai F, Chen C, Chao A. Hysteroscopic management of heterotopic cesarean scar pregnancy. Fertil Steril. 2010;94(4):1529. e15-8. http://dx.doi.org/10.1016/j.fertnstert.2010.02.039. PMid:20347078.

10. Litwicka K, Greco E, Prefumo F, Fratelli N, Scarselli F, Ferrero S, et al. Successful management of a triplet heterotopic caesarean scar pregnancy after in vitro fertilization-embryo transfer. Fertil Steril. 2011;95(1):291.e1-3. http://dx.doi.org/10.1016/j. fertnstert.2010.05.025. PMid:20646693.

11. Hsieh BC, Hwang JL, Pan HS, Huang SC, Chen CY, Chen PH. Heterotopic Caesarean scar pregnancy combined with intrauterine pregnancy successfully treated with embryo aspiration for selective embryo reduction: Case report. Hum Reprod. 2004;19(2):285-7. http://dx.doi.org/10.1093/humrep/deh080. PMid:14747168.

12. Demirel LC, Bodur H, Selam B, Lembet A, Ergin T. Laparoscopic management of heterotopic cesarean scar pregnancy with preservation of intrauterine gestation and delivery at term: case report. Fertil Steril. 2009;91(4):1293.e5-7. http://dx.doi.org/10.1016/j. fertnstert.2008.01.067. PMid:18353320.

13. Gupta R, Vaikousi E, Whitlow B. Heterotopic caesarean section scar pregnancy. J Obstet Gynaecol. 2010;30(6):626-7. http:// dx.doi.org/10.3109/01443615.2010.491565. PMid:20701516.

14. Bai XX, Gao HJ, Yang XF, Dong MY, Zhu YM. Expectant management of heterotopic cesarean scar pregnancy. Chin Med J (Engl). 2012;125(7):1341-4. PMid:22613612.

15. Uysal F, Uysal A. Spontaneous heterotopic cesarean scar pregnancy: conservative management by transvaginal sonographic guidance and successful pregnancy outcome. J Ultrasound Med. 2013;32(3):547-8. http://dx.doi.org/10.7863/jum.2013.32.3.547. PMid:23443198. 
16. Yazicioglu HF, Turgut S, Madazli R, Aygün M, Cebi Z, Sönmez S. An unusual case of heterotopic twin pregnancy managed successfully with selective feticide. Ultrasound Obstet Gynecol. 2004;23(6):626-7. http://dx.doi.org/10.1002/uog.1050. PMid:15170809.

17. Taşkin S, Taşkin EA, Ciftçi TT. Heterotopic cesarean scar pregnancy: how should it be managed? Obstet Gynecol Surv. 2009;64(10):690-5, quiz 697. http://dx.doi.org/10.1097/OGX.0b013e3181b8b144. PMid:19772678.

18. Dueñas-Garcia OF, Young C. Heterotopic cesarean scar pregnancy associated with a levonorgestrel-releasing intrauterine device. Int J Gynaecol Obstet. 2011;114(2):153-4. http://dx.doi.org/10.1016/j.ijgo.2011.02.021. PMid:21679948.

19. Ugurlucan FG, Bastu E, Dogan M, Kalelioglu I, Alanya S, Has R. Management of cesarean heterotopic pregnancy with transvaginal ultrasound-guided potassium chloride injection and gestational sac aspiration, and review of the literature. J Minim Invasive Gynecol. 2012;19(5):671-3. http://dx.doi.org/10.1016/j.jmig.2012.05.006. PMid:22935313.

20. Lui MW, Shek N, Li R, Chu F, Pun T. Management of heterotopic cesarean scar pregnancy by repeated transvaginal ultrasonographicguided aspiration with successful preservation of normal intrauterine pregnancy and complicated by arteriovenous malformation. Eur J Obstet Gynecol Reprod Biol. 2014;175:209-10. http://dx.doi.org/10.1016/j.ejogrb.2013.12.042. PMid:24491276.

21. Kim ML, Jun H, Kim J, Seong S, Cha D. Successful full-term twin deliveries in heterotopic cesarean scar pregnancy in a spontaneous cycle with expectant management. J Obstet Gynaecol Res. 2014;40(5):1415-9. http://dx.doi.org/10.1111/jog.12326. PMid:24690027.

22. Armbrust R, Krätschell R, Henrich W, David M. Operative therapy for heterotopic scar pregnancy and successful birth of the intrauterine foetus - case report and review of the literature. Geburtshilfe Frauenheilkd. 2015;75(4):384-8. http://dx.doi. org/10.1055/s-0035-1545918. PMid:26028696.

23. Abusheikha N, Salha O, Brinsden P. Extra-uterine pregnancy following assisted conception treatment. Hum Reprod Update. 2000;6(1):80-92. http://dx.doi.org/10.1093/humupd/6.1.80. PMid:10711833.

24. Fang C, Huang R, Wei L, Jia L. Frozen-thawed day 5 blastocyst transfer is associated with a lower risk of ectopic pregnancy than day 3 transfer and fresh transfer. Fertil Steril. 2015;103(3):655-661.e3. http://dx.doi.org/10.1016/j.fertnstert.2014.11.023. PMid:25542820.

25. Sahin S, Ozay A, Ergin E, Turkgeldi L, Kurum E, Ozornek H. The risk of ectopic pregnancy following GnRH agonist triggering compared with hCG triggering in GnRH antagonist ivf cycles. Arch Gynecol Obstet. 2015;291(1):185-91. http://dx.doi.org/10.1007/ s00404-014-3399-x. PMid:25078054.

\section{*Correspondence:}

Marcelo Lucchesi Montenegro

Fertway Fertility Clinic

EAv. Silva Jardim, 2103 - Água Verde,

CEP 80250-200, Curitiba, Parana, Brazil

(41) $99529-8899$

marcelolucmonte@gmail.com

\section{Author information}

MLM: Fertway Fertility Clinic (Curitiba, Parana, Brazil) Assistent Doctor. RF: Medical Student - Mackenzie Evangelical College of Paraná - Curitiba (FEMPAR). CWS: Fertway Fertility Clinic (Curitiba, Parana, Brazil) Assistent Doctor. FFF: Fertway Fertility Clinic (Curitiba, Parana, Brazil) Assistent Doctor.

\section{Author contributions}

MLM: responsible for gathering of data, literature review and article writing.

RF: responsible for table contents and figures edition; helped on literature review.

CWS: responsible for the case description and helped on literature review.

FFF: coordinate data analysis; responsible for patient management, procedures and therapeutic decisions. 